\title{
Avaliação da prematuridade em Hospital Amigo da Criança no interior de Sergipe
}

\section{no ano de 2019}

\author{
Evaluation of prematurity in a Baby-Friendly hospital in the interior of Sergipe in 2019 \\ Evaluación de la prematuridad en un Hospital Amigo da Criança en el interior de Sergipe en 2019
}

Recebido: 12/01/2022 | Revisado: 16/01/2022 | Aceito: 21/01/2022 | Publicado: 23/01/2022

\author{
Alexa Aragão do Sacramento \\ ORCID: https://orcid.org/0000-0002-0966-0393 \\ Hospital Santa Isabel, Brasil \\ E-mail: alexasacramento@hotmail.com \\ Izailza Matos Dantas Lopes \\ ORCID: https://orcid.org/0000-0001-9752-5628 \\ Hospital Santa Isabel, Brasil \\ E-mail: izailzamatos@ hotmail.com
}

\begin{abstract}
Resumo
A prematuridade é uma síndrome clínica complexa, com múltiplos fatores etiológicos, e está associada a um amplo espectro de condições clínicas correlacionadas à sobrevida e aos padrões de crescimento e desenvolvimento, definidos a partir do nascimento de crianças com idade gestacional menor do que 37 semanas. O presente estudo, de caráter epidemiológico, retrospectivo, transversal e de caráter analítico, objetivou identificar o perfil de mães e de prematuros nascidos vivos em uma maternidade no interior do estado de Sergipe. Os dados analisados foram obtidos por meio do sistema de informações sobre nascidos vivos (SINASC), com base nas declarações de nascidos vivos. Sua análise apontou que a maior parte das mães estava, respectivamente na faixa-etária de 20 a 24 anos (28,5\%); na de 25 a 28 anos $(27,3 \%)$; e na de 10 a 19 anos (20,2\%). No momento do nascimento das crianças, essas mulheres estavam em união consensual $(70,4 \%)$, ou eram casadas $(14,9 \%)$. Um grupo menor de mães registradas no SINASC era comporto por mulheres solteiras $(12,7 \%)$, separadas judicialmente, viúvas ou ignorado $(1,1 \%)(\mathrm{p}<0,05)$. No que tange ao prénatal, 68,5\% das mulheres tiveram um acompanhamento considerado mais do que adequado, com 7 ou mais consultas médicas $(\mathrm{p}<0,05)$. APGAR dos recém-nascidos (a maioria apresentou pontuação entre 8 a 10, no primeiro e no quinto minuto de vida $(\mathrm{p}<0,05)$. Os resultados da análise executada neste estudo sinalizaram a importância da identificação dos principais fatores de risco para o parto prematuro, e apontaram que esse quadro é menos frequente entre gestantes que mantém relação consensual ou são casadas. Por isso, um pré-natal adequado, bem como o conhecimento do perfil dessas mulheres, é de suma importância, pois desse modo é possível detectar as principais causas de parto prematuro e evitar o seu acontecimento.
\end{abstract}

Palavras-chave: Prematuridade; Pré-natal; Fatores de risco; Gestação.

\begin{abstract}
Prematurity, defined as a child born with a gestational age of fewer than 37 weeks, is a complex clinical syndrome with multiple etiological factors and is linked to a wide range of clinical conditions correlated with survival and pattern of growth and development. The study aimed to identify the profile of mothers and preterm infants born alive in a maternity hospital in the Sergipe's State interior. A retrospective, cross-sectional and epidemiological investigation was conducted. Data was acquired from the information system on live births (SINASC) based on the declarations of live births. Main findings: Maternal age group (28.5\% were between 20 and 24 years old, $27.3 \%$ between 25 and 29 years old, and 20.2\% were between 10 and 19 years old); Marital status (70.4\% of the mothers had a consensual union; $14.9 \%$ are married; 12.7 are single; the others are legally separated, widows or who ignored them, representing $1.1 \%(\mathrm{p}<0.05)$; Adequacy of prenatal care (most pregnancies had prenatal care classified as more than adequate, $68.5 \%$ of women had 7 or more prenatal consultations $(\mathrm{p}<0.05)$; APGAR of newborns ( most presented scores between 8 to 10 , in the first and fifth minutes of life $(\mathrm{p}<0.05))$. The data from this study highlights the importance of identifying the main risk factors for premature birth, reaffirming the importance of prenatal care started early. Furthermore, pregnant women who are in a consensual relationship or who are married have a lower rate of premature births, therefore knowing their profile is crucial. In conclusion, proper prenatal care, when started early, can assist in detecting and preventing the main reasons for premature birth.
\end{abstract}

Keywords: Prematurity; Prenatal; Risk-factors; Gestation.

\section{Resumen}

La prematuridad es un síndrome clínico complejo, con múltiples factores etiológicos, y se asocia a un amplio espectro de condiciones clínicas correlacionadas con la supervivencia y los patrones de crecimiento y desarrollo, definido a 
partir del nacimiento de niños con edad gestacional inferior a 37 semanas. El presente estudio, de carácter epidemiológico, retrospectivo, transversal y analítico, tuvo como objetivo identificar el perfil de las madres y los prematuros nacidos vivos en una maternidad del interior del estado de Sergipe. Los datos analizados se obtuvieron a través del sistema de información sobre nacimientos vivos (SINASC), basado en las declaraciones de nacimientos vivos. Su análisis señaló que la mayoría de las madres pertenecían, respectivamente, al grupo de edad de 20 a 24 años $(28,5 \%)$; de 25 a 28 años (27,3\%); y de 10 a 19 años (20,2\%). En el momento del nacimiento de los niños, estas mujeres estaban en una unión consensuada $(70,4 \%)$, o estaban casadas $(14,9 \%)$. Un grupo menor de madres registradas en el SINASC estaba compuesto por mujeres solteras $(12,7 \%)$, separadas legalmente, viudas o que las ignoraban $(1,1 \% \mathrm{p}<0,05)$. En cuanto a la atención prenatal, el 68,5\% de las mujeres tuvo un acompañamiento considerado más que adecuado, con 7 o más citas médicas $(\mathrm{p}<0,05)$. APGAR de los recién nacidos (la mayoría presentó puntuaciones entre 8 y 10, en el primer y quinto minuto de vida $(\mathrm{p}<0,05)$. Los resultados del análisis realizado en este estudio indicaron la importancia de la identificación de los principales factores de riesgo de parto prematuro, y señalaron que esta condición es menos frecuente entre las mujeres embarazadas que mantienen una relación consensuada o están casadas. Por eso, un adecuado control prenatal, así como el conocimiento del perfil de estas mujeres es de suma importancia, ya que así es posible detectar las principales causas de los partos prematuros y evitar que se produzcan.

Palabras clave: Prematuridad; Prenatal; Factores de riesgo; Embarazo.

\section{Introdução}

A prematuridade é uma síndrome clínica complexa, com múltiplos fatores etiológicos, e está associada a um amplo espectro de condições clínicas que definem a sobrevida e o padrão de crescimento e desenvolvimento das crianças, em diferentes subgrupos de risco. Inicia-se muito antes da gestação, sendo determinada pela relação entre fatores biológicos e socioeconômicos, como estilo de vida e de trabalho da mulher (Sociedade Brasileira de Pediatria, 2017).

Define-se a prematuridade pela idade gestacional em que os lactentes nascem. A criança nascida com idade gestacional menor que 37 semanas ou menos de 259 dias é denominada pré-termo. Quando ela nasce entre 34 e 36 semanas (e seis dias), é denominada pré-termo-tardio. Já a criança nascida de 23 a 33 semanas (e 6 dias) é considerada pré-termo moderado. A criança pode ainda ser muito pré-termo, quando nascida de 28 a 31 semanas (e seis dias), e pré-termo extremo, quando nascida com menos de 28 semanas. Apesar dos avanços na obstetrícia, o número de nascimentos prematuros ainda é elevado. Os fatores de risco podem ser divididos em epidemiológicos e obstétricos. Quanto aos epidemiológicos, os que se destacam são o baixo nível socioeconômico, gravidez nos extremos etários (< 15 anos e > 40 anos), desnutrição/dieta desequilibrada, baixo peso pré-gestacional, consumo de drogas ilícitas, estresse psicossocial, ausência de controle pré-natal. Dentre os fatores obstetrícios, os mais prevalentes são o histórico de partos prematuros anteriores, rotura prematura de membranas, infecção amniótica e geniturinárias, incompetência cervical, malformações fetais e placentárias, anomalias uterinas, gestação múltipla, trauma e cirurgias (Rodrigues \& Bolsoni-Silva, 2011).

No mundo, atualmente, a prematuridade é a causa de $75 \%$ dos casos de mortalidade neonatal. As prevalências de prematuridade no mundo, no Brasil e no Nordeste são de 9,2\%; 7,1\%; e 10,74\%, respectivamente (Almeida et al., 2012). Segundo Chawanpaiboon et al., (2019), comparado ao mundo, o Brasil ocupa o nono lugar em número absoluto de partos prematuros e precisamos prestar atenção nisso. O parto prematuro está relacionado a fatores de risco que podem ser prevenidos por um pré-natal de qualidade. Com a redução do número desses partos, diminui também os riscos de condições crônicas, alterações nos padrões de crescimento da criança desde o período neonatal, além de atrasos no desenvolvimento sensorial, motor e cognitivo e problemas psíquicos, com todas as consequências associadas a cada um desses eventos.

Diante do exposto, o objetivo deste trabalho é analisar os fatores de risco relacionados a prematuridade em uma maternidade na região Nordeste, no município de Itabaiana/SE. Os resultados serão encaminhados aos gestores envolvidos para que políticas públicas com o objetivo de reduzir os casos de prematuridade, o que pode ser feito a partir de uma melhor assistência materno-infantil. 


\section{Metodologia}

Este estudo possui um caráter observacional, com delineamento transversal, retrospectivo e analítico. Analisamos os dados de uma maternidade da região Nordeste, no município de Itabaiana/SE. Tais dados foram coletados por meio do SINASC, no item "Estatísticas Vitais", do programa TABNET, do Departamento de Informática do SUS (DATASUS), no período de 2019. As seguintes variáveis, relacionadas à genitora e ao recém-nascido, são contempladas: idade, escolaridade, estado civil, tipo de gravidez (única ou múltipla), via de parto (vaginal ou cesárea), realização de pré-natal (se adequado ou não), Idade gestacional (IG) do nascimento, APGAR no $1^{\circ}$ e no $5^{\circ}$ minuto, peso do nascimento. Como critérios de inclusão, consideramos crianças nascidas vivas no período de 2019, a partir dos registros no SINASC para a maternidade da região Nordeste, município de Itabaiana/SE. Aquelas que nasceram mortas ou abortadas foram excluídas do estudo.

Quanto aos aspectos éticos para a execução da pesquisa, o presente trabalho não foi submetido ao Comitê de Ética em Pesquisa (CEP), pois, segundo o inciso 3 do parágrafo único do Artigo $1^{\circ}$ da resolução nº510, de 07 de abril de 2016 , os dados foram extraídos de plataforma de domínio público, que não identificam os participantes da pesquisa, sendo liberados o TCLE e o TALE.

Para a codificação dos dados, consideramos variáveis qualitativas e quantitativas referentes à genitora (idade, escolaridade, estado civil, tipo de gravidez (única ou múltipla), via de parto (vaginal ou cesárea), realização de pré-natal (adequado, não adequado); e ao recém-nascido (idade gestacional do nascimento, APGAR no $1^{\circ}$ e no $5^{\circ}$ minuto, peso do nascimento). A partir dessas variáveis, executamos uma análise descritiva, para calcularmos valores absolutos e percentuais; e outra inferencial, para calcularmos a associação entre variáveis qualitativas, por meio do teste Qui-quadrado $\left(\chi^{2}\right)$ (Pearson, 1992). Quando a frequência observada foi menor que 5 utilizamos o teste Exato de Fisher (Fisher, 1922). Em todos os testes de hipótese realizados, a conclusão foi obtida através da interpretação do p-valor. Em todos os cruzamentos, foi adotado um nível de significância de $5 \%(\mathrm{p}<0,05)$. O software utilizado foi o R, versão 3.6.3.

\section{Resultados e Discussão}

Durante o período de execução deste estudo, durante o ano de 2019, ocorreram 4.211 nascimentos em Itabaiana, caracterizando um aumento de 6,3\% em relação ao ano anterior, no qual 3.960 nascimentos foram computados.

A faixa-etária materna de maior participação nos nascimentos foi a de 20 a 24 anos (28,5\%), seguida pela de 25 a 29 anos $(27,3 \%)$, e pela de 10 a 19 anos, conforme exposto no Gráfico 1:

Gráfico 1: Percentual de nascidos vivos segundo a idade das mães - Itabaiana - 2019.

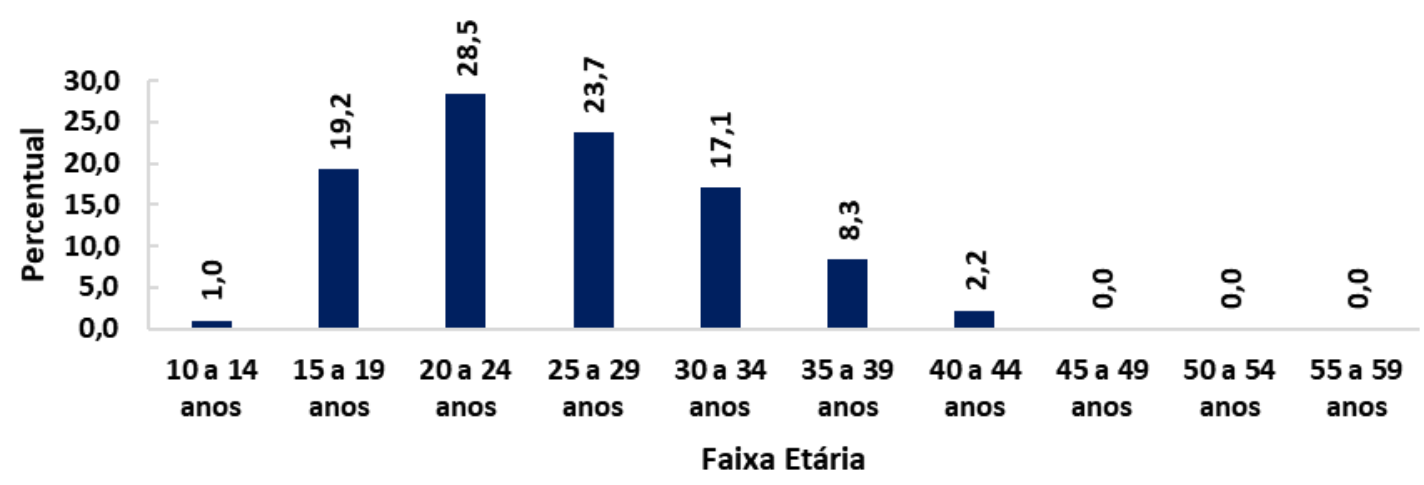

Fonte: DataSus.

A idade materna tem sido considerada condição de risco quando a gravidez ocorre nos extremos da vida reprodutiva, 
ou seja, quando essas gestações são precoces ou tardias (Cabral; Santos \& Cano, 2017). A gravidez na adolescência é o fator de maior concentração de agravos à saúde materna, bem como de complicações perinatais, tais como baixo ganho de peso materno, desproporção cefalopélvica, pré-eclâmpsia, prematuridade e baixo peso ao nascer. As intercorrências relativas à gravidez na adolescência podem ser potencializadas quando associadas a condições socioeconômicas desfavoráveis, bem como à fragilidade da estrutura familiar e dificuldade de acesso aos serviços assistenciais (Ramos \& Cuman, 2009).

Em relação à escolaridade, 53,2\% das mães têm entre 8 a 11 anos de estudos, 34,1\% estudaram de 4 a 7 anos, e $6,7 \%$ estudaram 12 anos ou mais, conforme disposto no gráfico 2 :

Gráfico 2: Percentual de nascidos vivos segundo a escolaridade das mães - Itabaiana - 2019.

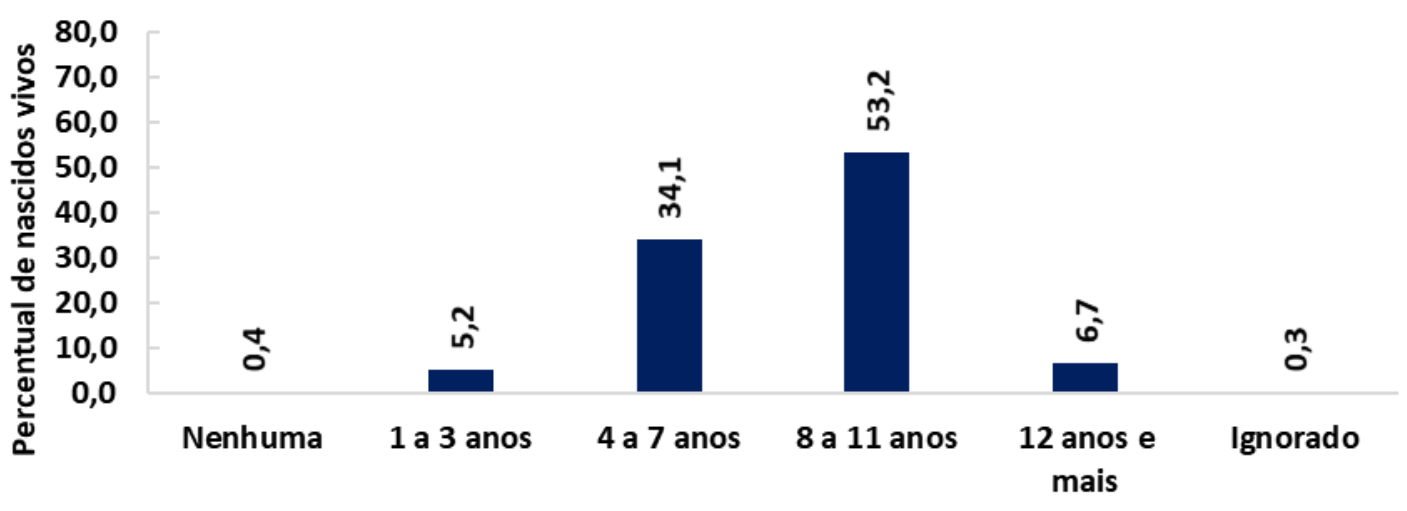

Anos de estudo

Fonte: DataSus.

Os resultados expostos no Gráfico 2 sugerem que o menor tempo de escolarização está relacionado à prematuridade, o que corrobora a literatura existente sobre esse tema. Em um estudo realizado em Quebec, no Canadá, as taxas de prematuridade diminuíram conforme o aumento da escolaridade. Entre mulheres com menos de 11 anos de estudo, o percentual de prematuridade foi de 7,9\%, e entre mulheres com mais de 17 anos de estudo, esse percentual foi de 4,9\% (Assunção et al., 2012). Outra pesquisa encontrou o risco para nascimento prematuro de $92 \%$ maior em mulheres com menor escolaridade quando comparadas com as de maior escolaridade (Santos et al., 2014). Desta forma, pode-se considerar escolaridade como uma variável determinada pelas condições socioeconômicas.

Quanto ao estado civil, dos nascimentos ocorridos, 70,4\% das mães tinham união consensual. Desse total, 14,9\% eram casadas; $12,7 \%$, solteiras, e as demais $(1,1 \%, \mathrm{p}<0,05)$ eram separadas judicialmente, viúvas ou foi ignorado, de acordo com os resultados expostos no quadro 3 . 
Gráfico 3: Percentual de nascidos vivos segundo o estado civil das mães - Itabaiana - 2019.

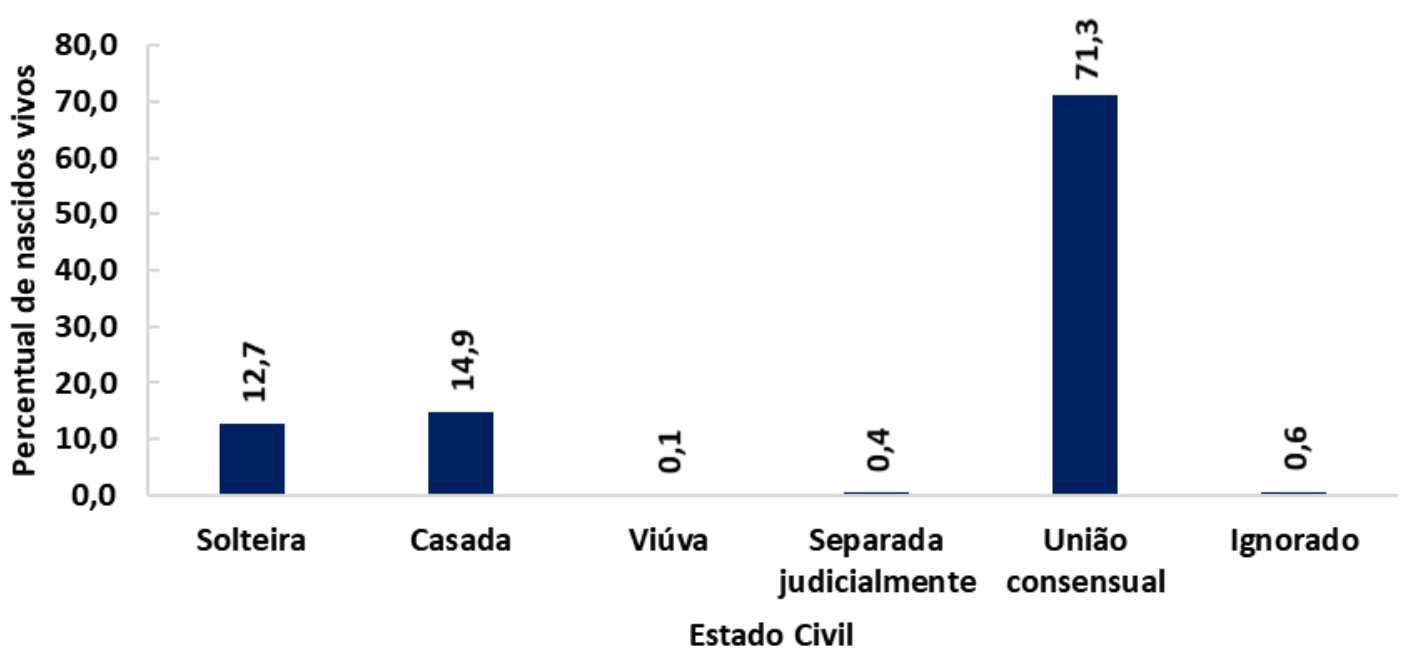

Fonte: DataSus.

Os resultados do presente estudo diferem da pesquisa realizada no município de Guarapuava/PR, que apontou uma prevalência de $>50 \%$ de gestantes solteiras, que não têm apoio de um companheiro e, portanto, não têm com quem dividir ou compartilhar suas dificuldades e responsabilidades (Ramos \& Cuman, 2009).

Analisando a adequação do pré-natal, observamos que a maioria das gestações teve a quantidade de consultas de prénatal classificadas como mais que adequada. $68,5 \%$ das mulheres realizaram 7 ou mais consultas de pré-natal, $25,9 \%$ realizaram de 4 a 6 consultas, 5,1\% realizaram de 1 a 3 consultas; e $0,5 \%$ não foram à nenhuma consulta (p< 0,05$)$. Os resultados que apresentamos sugerem que, apesar da padronização no serviço de saúde para a realização do acompanhamento do pré-natal, ainda existe a necessidade de maior adesão a esse protocolo, visto que o pré-natal, quando realizado adequadamente, é fator de grande importância para a prevenção das principais causas do parto prematuro.

Em relação às características da gestação e dos partos, houve predominância (95,6\% dos nascimentos) de recémnascidos a termo (37 a 41 semanas), o que era esperado, considerando que a maternidade de referência é de baixo risco. A maioria dos partos ocorridos no período que este trabalho abrange foi do tipo única (99,5\%). Todavia, esse fator não foi relevante para o presente estudo, como demonstrado no Gráfico 4.

Gráfico 4: Percentual de nascidos vivos segundo a idade gestacional - Itabaiana - 2019.

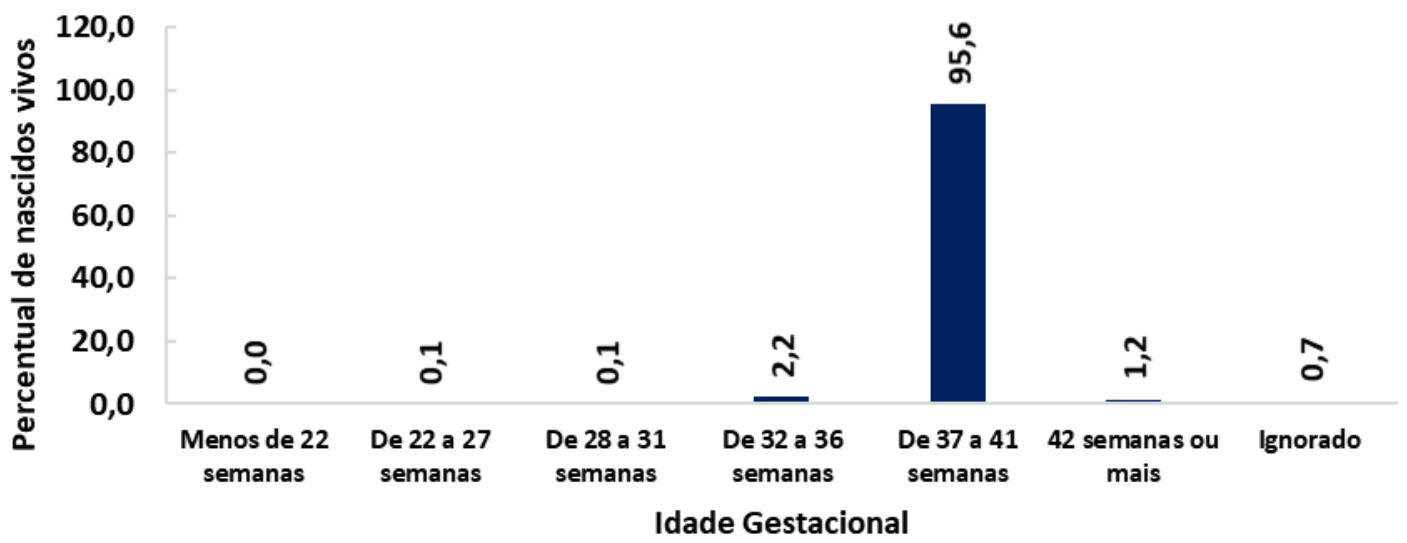

Fonte: DataSus. 
O percentual de partos vaginais $(63,2 \%)$ foi consideravelmente maior do que de partos cesáreos $(36,8 \%)$, conforme apresentado no Gráfico 5. Quando associado às variáveis, tanto faixa-etária/idade da genitora quanto faixa-etária/idade gestacional do nascimento obtiveram um $\mathrm{p}<0,05$.

Gráfico 5: Percentual do tipo de parto - Itabaiana - 2019.

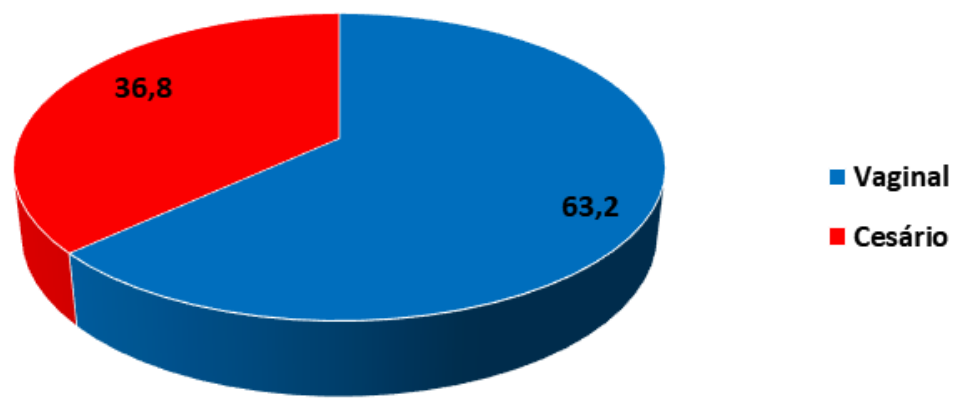

Fonte: DataSus.

Tais dados indicam que ainda há um percentual elevado de cesáreas, sendo esta intervenção muitas vezes desnecessária. Ocorre ainda uma desvalorização do parto normal, ocasionada também pela prática de intervenções cirúrgicas desnecessárias em grande número e frequência. Este é um indicativo da falta de informação e educação em saúde da população feminina, o que nos leva a crer que muitas mulheres não se sentem capazes de escolher seu parto quando confrontadas com a opinião técnica dos profissionais de saúde. Segundo a OMS a quantidade ideal de partos cesarianos é de 15\% em relação aos partos por via vaginal (Betran et al., 2015).

Em relação às características dos recém-nascidos, houve maior número de crianças com peso entre 3000 a $3999 \mathrm{~g}$ (69,1\%); com peso entre 2500 a $2999 \mathrm{~g}(21 \%) .6,2 \%$ pesavam entre 500 e $1499 \mathrm{~g}$ ( p < 0,05). Relacionando o peso de nascimento do recém-nascido com idade da gestante, infere-se que aquelas acima de 20 anos corresponderam a mais de $75 \%$ com filhos com peso adequado ao nascimento (2500 a $3999 \mathrm{~g}$ ), $\mathrm{p}<0,05$.

O estudo mostra que o baixo peso de recém nascidos está relacionado à prematuridade, o que caracteriza essa variável como fator de risco relacionado à morbimortalidade neonatal. Os resultados de um estudo realizado em Santa Maria apontaram que $78 \%$ dos bebês com baixo peso nasceram prematuros, sendo a prematuridade a principal responsável pelo baixo peso ao nascer (BP) (Rades et al., 2004).

A OMS define como BP ao nascer o recém-nascido com menos de $2.500 \mathrm{~g}$, independentemente da idade gestacional (WHO, 2014). Este é um fator importante para determinar a desnutrição, pois reflete as condições nutricionais tanto do recémnascido quanto da gestante; influencia o crescimento e desenvolvimento da criança e, a longo prazo, pode repercutir nas condições de saúde do adulto (Motta et al., 2005).

Relacionando a duração da gestação com APGAR dos recém-nascidos, observamos que a maioria apresentou pontuação entre 8 a 10 no primeiro e no quinto minuto de vida, com 92\% e 97,2\%, respectivamente. Porém, o APGAR < 7 prevaleceu entre os recém-nascidos com IG $<36$ semanas ( $p<0,05$ ), como mostra os gráficos 6 e 7. 
Gráfico 6: Percentual de nascidos vivos segundo o apgar $1^{\circ}$ minuto- Itabaiana - 2019.

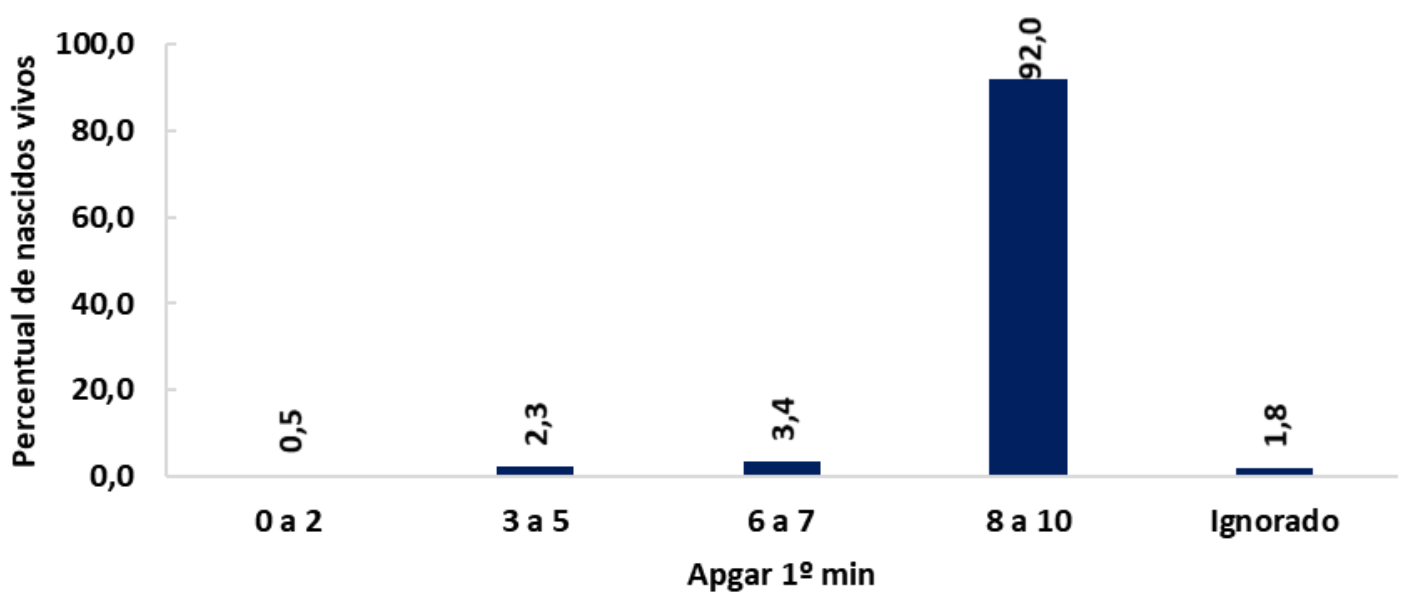

Fonte: DataSus.

Gráfico 7: Percentual de nascidos vivos segundo o apgar 5º minuto- Itabaiana - 2019.

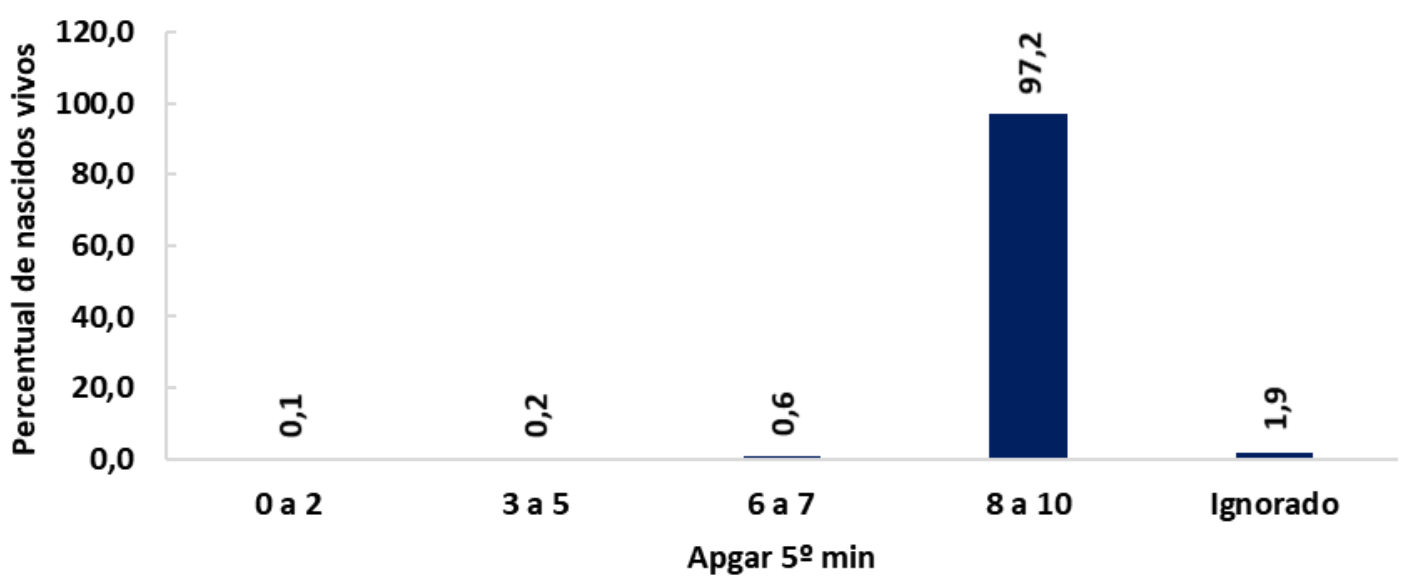

Fonte: DataSus.

O índice de APGAR baixo é útil para identificar os recém-nascidos que necessitam de cuidados adicionais. APGAR de 7 a 10 significa que o bebê é sadio e que provavelmente não terá problemas futuros. APGAR inferior a 7 é sinal de alerta de acordo com a alteração fisiopatológica e da maturidade do concepto (Oliveira et al., 2012).

Os resultados apresentados na Tabela 1 sugerem que a realização de 7 ou mais consultas de pré-natal e sua adequação estão associadas ao nascimento de bebês com idade gestacional entre 37 e 41 semanas e a prematuridade. A ausência de cuidados pré-natais está associada a um aumento do risco de baixo peso ao nascer, partos prematuros e mortalidade materna e infantil (Parker et al., 2001).

Em 73,1\% dos casos, os partos vaginais estão relacionados à prematuridade, resultado diferente daqueles apresentados no estudo realizado em 2012, em Porto Alegre/Rio Grande do Sul. Nesse estado, a cesariana apresentou maior proporção de nascimentos prematuros, e os recém-nascidos por cesariana tiveram 15\% a mais de chances de serem prematuros (Oliveira et al., 2016).

O peso ao nascer dos prematuros foi, em $56,7 \%$ dos casos, acima de $2500 \mathrm{~g}$, sendo este um dado positivo. Em um estudo do tipo transversal realizado em Santa Maria, evidenciou-se que 78\% dos bebês com baixo peso nasceram prematuros (Rades et al., 2004). Observou-se ainda que 75,3\% e 94,8\% deles tinham, respectivamente, APGAR entre 8 e 10 no primeiro e 
quinto minuto de vida. Este dado também é positivo, visto que o APGAR no $5^{\circ}$ minuto $<7$ pode ser considerado indicador de asfixia, o que aumenta o risco de morte (Oliveira et al., 2012). Logo, pode-se dizer que um escore menor que 7 é um sinal de alerta para atenção maior (Silva et al., 2020).

Tabela 1 - Associação entre Idade Gestacional (IG) do nascimento e demais variáveis.

\begin{tabular}{|c|c|c|c|c|}
\hline \multirow{2}{*}{ Variável/Categoria } & \multicolumn{3}{|c|}{ Duração gestação (semanas) } & \multirow{2}{*}{ P-valor } \\
\hline & Até 36 & De 37 a 41 & 42 ou mais & \\
\hline \multicolumn{5}{|l|}{ Consultas pré-natais } \\
\hline Até 3 consultas & $16(15,4)$ & $209(5,2)$ & $5(9,8)$ & 0,0000 \\
\hline De 4 a 6 consultas & $40(38,5)$ & $1026(25,5)$ & $19(37,3)$ & \\
\hline 7 ou mais consultas & $48(46,2)$ & $2790(69,3)$ & $27(52,9)$ & \\
\hline \multicolumn{5}{|l|}{ Adequação do pré-natal } \\
\hline Não fez / Inadequado & $21(21,2)$ & $673(16,8)$ & $17(34,0)$ & 0,0000 \\
\hline Intermediário & $20(20,2)$ & $394(9,8)$ & $8(16,0)$ & \\
\hline Adequado/mais que adequado & $58(58,6)$ & $2947(73,4)$ & $25(50,0)$ & \\
\hline \multicolumn{5}{|l|}{ Tipo de parto } \\
\hline Vaginal & $76(73,1)$ & $2533(62,9)$ & $32(72,7)$ & 0,0451 \\
\hline Cesário & $28(26,9)$ & $1492(37,1)$ & $12(27,3)$ & \\
\hline \multicolumn{5}{|l|}{ Peso ao nascer } \\
\hline Até 2499g & $44(42,3)$ & $110(2,8)$ & $0(0,0)$ & 0,0000 \\
\hline 2500g a 3999g & $59(56,7)$ & $3665(91,1)$ & $45(88,2)$ & \\
\hline $4000 \mathrm{~g}$ e mais & $1(1,0)$ & $250(6,2)$ & $6(11,8)$ & \\
\hline \multicolumn{5}{|l|}{ Apgar $1^{\circ}$ minuto } \\
\hline 0 a 2 & $4(4,1)$ & $18(0,5)$ & $0(0,0)$ & 0,0000 \\
\hline 3 a 5 & $5(5,2)$ & $88(2,2)$ & $2(4,0)$ & \\
\hline 6 a 7 & $15(15,5)$ & $121(3,1)$ & $6(12,0)$ & \\
\hline 8 a 10 & $73(75,3)$ & $3734(94,3)$ & $42(84,0)$ & \\
\hline \multicolumn{5}{|l|}{ Apgar $5^{\circ}$ minuto } \\
\hline 0 a 2 & $3(3,1)$ & $2(0,1)$ & $0(0,0)$ & 0,0006 \\
\hline 3 a 5 & $1(1,0)$ & $7(0,2)$ & $0(0,0)$ & \\
\hline 6 a 7 & $1(1,0)$ & $26(0,7)$ & $0(0,0)$ & \\
\hline 8 a 10 & $91(94,8)$ & $3922(99,1)$ & $50(100,0)$ & \\
\hline
\end{tabular}

Fonte: Autores.

Quanto à Tabela 2, verifica-se que as gestantes mais jovens (com idade entre 10 e 29 anos) com o estado civil solteira, realizaram menos consultas de pré-natal e foram classificadas com pré-natal inadequado. Em um estudo correlacionando a relação conjugal genitora, a mortalidade infantil e aspectos socioeconômicos demonstrou que a taxa de mortalidade infantil é maior para crianças de mães solteiras, exceto as de raça branca, que têm alto grau de instrução (Ramos \& Cumann, 2009). Tais resultados sugerem que essas gestantes necessitam de mais apoio e de estratégias para incentivar o início precoce do pré-natal, a fim de prevenir complicações futuras. É possível que essas gestantes possam estar despreparadas para serem mães, vivenciando ansiedade permeada frente ao período gravídico, visto que poderão enfrentar mudanças no contexto social e familiar (Rosa et al., 2021).

A adolescência também está relacionada a maior quantidade de partos vaginas e nascimento de bebês com menos de 2500g, como mostra na Tabela 2, pois a idade materna exerce influência na ocorrência de parto prematuro. Mulheres adolescentes (menores de 20 anos) e com idade avançada (maiores de 34 anos) apresentaram associação com o parto prematuro (Gaiva et al., 2014; Newburn-Cook \& Onvskiw, 2005). Além disso, a não aceitação da gravidez associou-se ao início da APN tardio e ao número de consultas inferior a seis, confirmando os achados de outras pesquisas que sugerem que a gravidez indesejada é associada ao cuidado pré-natal inadequado (Belfort et al., 2018). É necessário preocupar-se também com 
os recém-nascidos prematuros e suas condições perinatais, bem como os agravos no desenvolvimento e crescimento infantil (Rosa et al., 2020). Neste sentido o estudo de Rosa, et al. (2021) conclui ser necessário mudanças nas práticas obstétricas para orientar o desenvolvimento de medidas efetivas para melhorar a qualidade de vida de prematuros moderados e tardios, promover promoção a saúde e prevenção do desenvolvimento dos agravos agudos de saúde.

Tabela 2 - Associação entre Idade da mãe e demais variáveis.

\begin{tabular}{|c|c|c|c|c|c|}
\hline \multirow{2}{*}{ Variável/Categoria } & \multicolumn{4}{|c|}{ Idade da mãe (anos) } & \multirow[t]{2}{*}{ P-valor } \\
\hline & 10 a 19 & 20 a 29 & 30 a 34 & 35 a 49 & \\
\hline \multicolumn{6}{|l|}{ Estado Civil } \\
\hline Solteira & $140(16,5)$ & $280(12,8)$ & $70(9,8)$ & $46(10,5)$ & 0,0000 \\
\hline Casada & $21(2,5)$ & $301(13,8)$ & $177(24,8)$ & $128(29,2)$ & \\
\hline União consensual & $687(80,9)$ & $1594(73,0)$ & $461(64,7)$ & $258(58,9)$ & \\
\hline Outras & $1(0,1)$ & $10(0,5)$ & $5(0,7)$ & $6(1,4)$ & \\
\hline \multicolumn{6}{|l|}{ Consultas Pré-Natais } \\
\hline Até 3 consultas & $61(7,2)$ & $101(4,6)$ & $38(5,3)$ & $37(8,4)$ & 0,0002 \\
\hline De 4 a 6 consultas & $243(28,6)$ & $551(25,1)$ & $167(23,3)$ & $128(28,9)$ & \\
\hline 7 ou mais consultas & $547(64,3)$ & $1546(70,3)$ & $513(71,4)$ & $278(62,8)$ & \\
\hline \multicolumn{6}{|l|}{ Adequação do Pré-Natal } \\
\hline Não fez/ inadequado & $191(22,6)$ & $353(16,1)$ & $99(13,9)$ & $71(16,1)$ & 0,0000 \\
\hline Intermediário & $81(9,6)$ & $218(9,9)$ & $66(9,2)$ & $60(13,6)$ & \\
\hline Adequado & $76(9,0)$ & $178(8,1)$ & $68(9,5)$ & $44(10,0)$ & \\
\hline Mais que adequado & $496(58,8)$ & $1442(65,8)$ & $481(67,4)$ & $265(60,2)$ & \\
\hline \multicolumn{6}{|l|}{ Tipo de parto } \\
\hline Vaginal & $615(72,3)$ & $1410(64,1)$ & $402(56,0)$ & $232(52,4)$ & 0,0000 \\
\hline Cesário & $236(27,7)$ & $788(35,9)$ & $316(44,0)$ & $211(47,6)$ & \\
\hline \multicolumn{6}{|l|}{ Peso ao nascer } \\
\hline Até 2499 g & $52(6,1)$ & $66(3,0)$ & $26(3,6)$ & $13(2,9)$ & 0,0006 \\
\hline 2500 g a 3999 g & $759(89,2)$ & $1991(90,6)$ & $650(90,5)$ & $393(88,7)$ & \\
\hline 4000 g e mais & $40(4,7)$ & $141(6,4)$ & $42(5,8)$ & $37(8,4)$ & \\
\hline
\end{tabular}

Fonte: Autores.

\section{Considerações Finais}

Os resultados do presente estudo sinalizam a importância da identificação dos principais fatores de risco para o parto prematuro, e reafirmam a importância de um pré-natal de qualidade, iniciado precocemente.

O pré-natal tem um papel fundamental no combate aos nascimentos prematuros, por isso é fundamental que haja a captação precoce da gestante sua realização. Através dele, é possível identificar os riscos gestacionais para o nascimento prematuro e tratar eventuais intercorrências para que, caso seja necessário, a mãe e a criança sejam encaminhadas para serviços de maior complexidade.

A etiologia do parto prematuro é multifatorial, constituindo-se como um problema de saúde pública complexo. Levase em consideração a preocupação constante com os recém-nascidos prematuros assim como com as condições perinatais que repercutem sobre eles. A fragilidade dos recém-nascidos prematuros contribui para a possibilidade de riscos, agravos e sequelas de diversos tipos, com diferentes consequências no processo de crescimento e desenvolvimento infantil. Portanto, é essencial prever e considerar riscos e prognósticos para que se possa eventualmente instituir medidas preventivas.

Diante do importante papel da prematuridade na mortalidade infantil, é imperioso que as pesquisas voltadas para esse tema tenham continuidade para elucidar as principais causas preveníveis da prematuridade, com o intuito de auxiliar no 
planejamento de ações preventivas assim como o seu combate, diminuindo, consequentemente, a morbimortalidade infantil.

As causas preveníveis mais frequentes do parto prematuro estão relacionadas à qualidade do pré-natal. Pesquisas futuras devem relacionar os principais fatores de risco regionalmente e a partir desses resultados implementar melhorias na assistência pré-natal e assim reduzir a morbidade e a mortalidade infantil no estado. O nascimento de parto prematuro gera desequilíbrio financeiro para a saúde pública e emocional para a família. O melhor tratamento da prematuridade é não deixar ela aconteça.

\section{Referências}

Almeida, A. C., Jesus, A. C. P., Lima, P. F. T., Araújo, M. F. M., \& Araújo, T. M. (2012). Fatores de risco maternos para prematuridade em uma maternidade pública de Imperatriz-MA. Revista Gaúcha de Enfermagem, 33(2),86-94.

Assunção, P. L., Novaes, H. M. D., Alencar, G. P., Melo, A. S. O., \& Almeida, M. F. (2021). Fatores associados ao nascimento pré-termo em Campina Grande, Paraíba, Brasil: um estudo caso-controle. Caderno de Saúde Pública, 28(6), 1078-1090.

Belfort, G. P., Santos, M. M. A. S., Pessoa, L. S., Dias, J. R., Heidelmann, S. P., \& Saunders, C. (2018). Determinantes do baixo peso ao nascer em filhos de adolescentes: uma análise hierarquizada. Ciência \& Saúde Coletiva, 23(8), 2609-2620.

Betran, A. P., Torloni, M. R., Zhang, J., Ye, J., Mikolajczyk, R., Deneux-Tharaux, C., Oladapo, O. T., Souza, J. P., Tunçalp, Ö., Vogel, J. P., \& Gülmezoglu, A. M. (2015). What is the optimal rate of caesarean section at population level? A systematic review of ecologic studies. Reproductive Health, 12(1), 1-10.

Cabral, R. A., Santos, B. M. O. \& Cano, M. A. T. (2017). A experiência de ser mãe pela primeira vez após os 35 anos. Revista Cinergis, 18(4), $279-284$.

Chawanpaiboon, S., Vogel, J. P., Moller, A. B., Lumbiganon, P., Petzold, M., Hogan, D., Landoulsi, S., Jampathong, N., Kongwattanakul, K., Laopaiboon, M., Lews, C., Rattanakanokchai, S., Teng, D. N., Thinkhamrop, J., Watananiriun, K., Zhang J., Zhou, W., \& Gulmezoglu, A. M. (2019). Global, regional, and national estimates of levels of preterm birth in 2014: a systematic review and modelling analysis. Lancet Glob Health,7(1), 37 - 46.

Fisher, R. A. (1922). On the Interpretation of $\mathrm{x}^{2}$ from Contingency Tables, and the Calculation of P. Journal of the Royal Statistical Society, 85(1), 87-94.

Gaiva, M. A., Fujimori, E., \& Sato, A. P. (2012). Pediatric patient classification system: improvement of an instrument. Revista da Escola de Enfermagem da USP, 48(5), 778-786.

Motta, M. E., Silva, G. A., Araújo, O. C., Lira, P. I., \& Lima, M. C. (2005). O peso ao nascer influencia o estado nutricional ao final do primeiro ano de vida?. Jornal de Pediatria, 81(5), 377- 382.

Newburn-Cook, C. V., \& Onyskiw, J. E. (2005). Is older maternal age a risk factor for preterm birth and fetal growth restriction? A systematic review. Health Care for Women International, 26(9), 852-875.

Oliveira, L. L., Gonçalves, A. C., Costa, J. S. D., Bonilha, A. L. L. (2016). Maternal and neonatal factors related to prematurity. Revista da Escola de Enfermagem da USP, 50(3), 382-389.

Oliveira, T. G., Freire, P. V., Moreira, F. T., Moraes, J. S., Arrelaro, R. C., Rossi, S. Ricardi, V. A., Juliano, Y, Novo, N. F., \& Bertagnon, J. R. (2012). Escore de Apgar e mortalidade neonatal em um hospital localizado na zona sul do município de São Paulo. Einstein, 10(1), 22-28.

Parker, J. D., Schoendorf, K. C., \& Kiely, J. L. (2001). A comparison of recent trends in infant mortality among twins and singletons. Paediatric and Perinatal Epidemiology, 1(15), 12-18.

Pearson, K. (1992). X. On the criterion that a given system of deviations from the probable in the case of a correlated system of variables is such that it can be reasonably supposed to have arisen from random sampling. The London, Edinburgh, and Dublin Philosophical Magazine and Journal of Science, 50(5), 157175 .

Rades, E., Bittar, R. E., \& Zubaig, M. (2004). Determinantes diretos do parto prematuro eletivo e os resultados neonatais. Revista Brasileira de Ginecologia e Obstetricia, 26(8), 655-662.

Ramos, H. A. C., \& Cuman, R. K. N. (2009). Fatores de risco para prematuridade: pesquisa documental. Escola Anna Nery - Revista de Enfermagem, 13(2), 297-304.

Rodrigues O. M. P. R., \& Bolsoni-Silva, A. T. (2011) Efeitos da prematuridade sobre o desenvolvimento de lactentes. Revista Brasileira de Crescimento e Desenvolvimento Humano, 21(1), 111-121.

Rosa, N. P., Mistura, C., Leivas, D. V. P., Veiga, T. M., Neves, E. T., \& Pereira, L. D. (2021). Risk factors and causes related to newborn prematurity in a hospital institution. Research, Society and Development, 10(9), e55610918431.

Rosa, N. P., Oliveira, D. C., Jantsch, L. B. \& Neves, E. T. (2020). Moderate and late previous pregnant baby health accidents in the neonatal period. Research, Society and Development, 9(7), e251974156.

Santos, N. L., Costa, M. C., Amaral, M. T., Vieira, G. O., Bacelar, E. B., \& Almeida, A. H. (2014). Gravidez na adolescência: análise de fatores de risco para baixo peso, prematuridade e cesariana. Ciência e Saúde Coletiva, 19(3), 719-726. 
Research, Society and Development, v. 11, n. 2, e20711225637, 2022

(CC BY 4.0) | ISSN 2525-3409 | DOI: http://dx.doi.org/10.33448/rsd-v11i2.25637

Silva, L. S. R., Cavalcante, A. N., Carneiro, J. K. R. \& Oliveira, M. A. S. (2020). Índice de Apgar correlacionado a fatores maternos, obstétricos e neonatais a partir de dados coletados no Centro de Saúde da Família do bairro Dom Expedito Lopes situado no município de Sobral/CE. Revista Científica da Faculdade de Medicina de Campos, 15(1), 25-30.

Sociedade Brasileira de Pediatria. (2017). Prevenção da prematuridade - uma intervenção da gestão e da assistência. Departamento Científico de Neonatologia, 2, 1-4.

WHO - World Health Organization. (2014). Global Nutrition Targets 2025 Low Birth Weight Policy Brief. https://apps.who.int/iris/handle/10665/149020. 